mutations of $P L A 2 G 6$, in addition, suggests that the fetal NAD is a new entity, distinct from INAD, with different molecular basis. Associated malformations suggest a wide phenotypic spectrum and probable genetic heterogeneity. Finally, fetal NAD is an additional etiology of fetal akinesia.

\section{Abstract A8}

Motor neuron disease presenting with fetal akinesia

P. Shannon ${ }^{1}$, D. Chitayat ${ }^{1}$, K. Chong ${ }^{1}$, C. Dunham ${ }^{2}$, C. Fallet-Bianco ${ }^{3}$

${ }^{1}$ Mount Sinai Hospital, Toronto; ${ }^{2}$ British Columbia Children's Hospital, Vancouver; ${ }^{3} \mathrm{CHU}$ Sainte-Justine, Montreal

doi:10.1017/cjn.2018.44

By contrast to infantile spinal muscular atrophy, which usually links to deletions in the SMN genes, fetal onset motor neuron disease is poorly reported. We collected a series of twelve cases of fetal arthrogryposis (16-31 weeks gestational age) with fetal motor neuron disease and excluded infectious diseases, lysosomal storage disease and neuroaxonal dystrophy. Of these twelve, 3 were thought to be ischemic in nature with microvascular alterations and systemic or central nervous system ischemic injury. The remaining 9 all displayed marked reduction in anterior horn motor neurons. Of these 9 , four demonstrated mineralised neurons, four demonstrated either neuronal loss or cavitation in the globus pallidus, and in two, degenerating neurons were detectable in the brainstem or globus pallidus. Specific sequencing of SMN1 was performed in 6 of 9 and was reported as normal. Whole exome sequencing was performed in 4 without definitive diagnosis. We conclude that fetal motor neuron disease can be distinguished from ischemic injury, is morphologically heterogeneous, may affect the globus pallidus and is rarely linked to SMN1 mutations.

\section{Abstract A9}

\section{The central nervous system lesion in amniotic rupture sequence}

\section{P. Shannon}

Mt Sinai Hospital, Toronto, Ontario

doi:10.1017/cjn.2018.45

We review the central nervous system anatomy in nine cases of amniotic rupture sequence, all of which had neuropathological examinations. Of these, four had normal brains, and in none of these was the cranial vault involved, and one had cleft lip and palate. Of the remaining five, all had portions of the scalp, calvarium and dura replaced by amnionic membrane directly overlying arachnoid. In one, the membrane covered a narrow necked large encephalocele, and the contained brain demonstrated extensive disruption and degeneration. In the remaining four, one demonstrated cranioplacental adhesion, and in three there was a broad based encephalocele covered in large part by amnion. Two of these four cases demonstrated holoprosencephaly. One case with holoprosencephaly and one without demonstrated marked aqueductal stenosis, and two of the four demonstrated aqueductal occlusion or near occlusion by neuroglial excrescences. None demonstrated ventriculomegaly. Three of these four cases demonstrate varying degrees of mechanical distortion and secondary pathology. We conclude that brains with amnionic rupture sequence demonstrate both malformation and deformation, which likely points to the embryonic stage origin of the lesion.

\section{Abstract A10}

Chronic traumatic encephalopathy in contact sports: The Canadian experience

\section{L.N. Hazrati}

University of Toronto, Toronto, Ontario, Canada

doi:10.1017/cjn.2018.46

Chronic traumatic encephalopathy (CTE) is suggested to be a progressive neurodegenerative disease, characterized by tau deposits in the depth of cortical sulci in neurons and in glioneuronal complexes around blood vessels. Few studies have suggested that it is caused by multiple concussions or subconcussive brain injuries. A recent publication showed that most American football players whose brain were donated to the Boston University concussion center had CTE (Mez et al. 2017). Over the last 6 years, with the help of neuropathologist colleagues across Canada, we have collected the brains of 33 high level professional and amateur athletes. These include 5 National hockey league (NHL) players, 15 Canadian football league (CFL) players, 3 College football players, 3 College hockey players, 2 professional boxers, 1 professional bull rider, 1 BMX champion, 1 rugby player and 2 skiers. All were male and the ages ranging from 15 to 87 years. Our results indicate that only a small portion of cases have CTE. Moreover, most cases are low stage (stage 1 or 2) and this pathology is mainly seen in some of the younger players. Older players either have no pathological findings or have other neurodegenerative diseases such as Alzheimer's disease. The disparity of results between the 2 groups will be discussed.

\section{Abstract A11}

Executive dysfunction and altered cerebrovascular activity in a rodent model of vascular cognitive impairment

K.D. Langdon ${ }^{1}$, C. Cordova ${ }^{2}$, S. Granter-Button ${ }^{2}$, J. Boyd $^{3}$, J. Peeling ${ }^{4}$, T. Murphy ${ }^{3}$, D. Corbett ${ }^{5}$

${ }^{1}$ Department of Pathology and Laboratory Medicine, Western University; ${ }^{2}$ Memorial University, Faculty of Medicine; ${ }^{3}$ University of British Columbia, Psychiatry; ${ }^{4}$ University of Manitoba College of Medicine, Radiology; ${ }^{5}$ University of Ottawa, Department of Cellular and Molecular Medicine

doi:10.1017/cjn.2018.47

Most basic science research has focused on overt stroke caused by blockage of major blood vessels. Less attention has been paid to small vessel disease giving rise to covert stroke that often leads to vascular cognitive impairment (VCI). One reason for this may be the relative lack of relevant animal models. This talk will describe a model of VCI induced in middle-aged SpragueDawley rats exposed to a diet high in saturated fats, salt and refined sugar (HFSS). In Experiment 1, rats fed HFSS and subjected to a small mediodorsal (MD) thalamic stroke with or without concomitant cerebral hypoperfusion experienced significant executive dysfunction. In Experiment 2, dietary 